\title{
BMJ Open Cohort profile: the diabetes-tuberculosis treatment outcome (DITTO) study in Pakistan
}

\author{
Fatima Mukhtar, ${ }^{1,2}$ Zahid A Butt ${ }^{3,4}$
}

To cite: Mukhtar F, Butt ZA. Cohort profile: the diabetestuberculosis treatment outcome (DITTO) study in Pakistan. BMJ Open 2016;6:e12970.

doi:10.1136/bmjopen-2016012970

- Prepublication history for this paper is available online. To view these files please visit the journal online (http://dx.doi.org/10.1136/ bmjopen-2016-012970).

Received 8 June 2016 Revised 4 November 2016 Accepted 9 November 2016

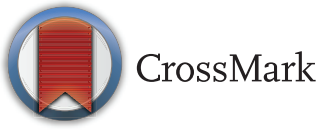

${ }^{1}$ Health Services Academy, Islamabad, Pakistan

${ }^{2}$ Department of Community Medicine, Lahore Medical \& Dental College, Lahore, Pakistan

${ }^{3}$ Department of Epidemiology \& Biostatistics, Health Services Academy, Islamabad, Pakistan ${ }^{4}$ School of Population and Public Health, University of British Columbia, Vancouver, British Columbia, Canada

Correspondence to Dr Fatima Mukhtar; fatimamukhtar@doctor.com

\section{ABSTRACT}

Purpose: Pakistan is faced with an increasing prevalence of diabetes in addition to its existing high burden of tuberculosis (TB). Diabetes has a detrimental effect on treatment outcomes of patients with TB, which may hinder achieving the goals of the End-TB strategy by 2030 . We conducted a prospective cohort study to determine difference between treatment outcomes among patients with diabetes and new pulmonary tuberculosis (PTB) and patients without diabetes and new PTB. This would help generate contextual and valid scientific evidence from a developing country like Pakistan with its unique interplay of sociocultural, economic and health system factors to inform policy and practice.

Participants: This paper outlines the baseline characteristics of 614 new cases of PTB, aged 15 years and older, which were followed up prospectively at 2nd, 5th and 6th months while on antituberculosis treatment and at 6 months after treatment completion.

Findings to date: We ascertained patients' diabetic status by conducting random and fasting blood glucose tests and their glycaemic control by determining glycosylated haemoglobin. Treatment outcomes were established using standardised definitions provided by WHO. The assessment of 614 respondents' diabetic status revealed that 113 (18\%) were diabetic and 501 (82\%) were nondiabetic. A greater proportion of patients with diabetes and PTB were illiterate $(n=74 / 113,65.5 \%)$ as compared to patients without diabetes and PTB $(n=249 / 501,50 \%)(p=0.035)$. More patients with diabetes and PTB gave a history of heart disease $(n=14 / 113,12 \%)$ and hypertension $(n=26 / 113$, $23 \%$ ) as compared to patients without diabetes and PTB ( $n=2 / 501,0.4 \%$ (heart disease) and $n=13501$, $3 \%$ (hypertension)) $(p<0.001)$. Unfavourable treatment outcome was more likely among patients with diabetes and PTB ( $n=23 / 93,25 \%)$ as opposed to patients without diabetes and PTB $(n=46 / 410$, $11 \%)(p=0.001)$.

Future plans: We are negotiating with the government regarding funding for a further 2-year follow-up of the cohort to ascertain death and relapse in the post-treatment period and also differentiate between re-infection and recurrence among these patients with respect to their diabetic status.

\section{Strengths and limitations of this study}

- The diabetes-tuberculosis treatment outcome (DITTO) study is the first prospective cohort study which has been conducted in Pakistan to determine the effect of diabetes on the treatment outcome of patients with tuberculosis.

- The ascertainment of diabetic status of DITTO cohort was based on two tests; one random and the other fasting blood glucose test.

- The main limitations are the inability to conduct drug susceptibility testing of patients and to determine their HIV status at the time of recruitment into the DITTO cohort due to nonavailability of funds.

\section{INTRODUCTION}

There is a resurgence of interest in the dual epidemic of diabetes and tuberculosis (TB) with the global increase in the diabetic population. This converging epidemic of diabetes and TB has many untoward and detrimental effects. ${ }^{1}$ The risk of development of $\mathrm{TB}$ is tripled by diabetes. ${ }^{2}$ It increases the severity related to $\mathrm{TB}$ and also slows the response to TB treatment. ${ }^{3}$ The clearance of Mycobacterium tuberculosis from the sputum, which is required to declare the patient noninfectious, is also delayed by diabetes. ${ }^{4}$ Diabetes increases the risk of treatment failure, death and relapse among patients with $\mathrm{TB}$, which poses a challenge for the developed and developing world. ${ }^{15}$

Low-income and middle-income countries such as Pakistan are likely to be affected the most as they are struggling with their existing high burden of TB and the growing population of diabetics. Pakistan ranks fourth in terms of global burden of TB with 630000 cases and an estimated incidence of 231 cases per 100000 population. ${ }^{6} 7$ Pakistan is one of the 10 countries with the highest number of patients with diabetes and a prevalence ranging from $7.6 \%$ to $11 \% .^{8}{ }^{9} \mathrm{It}$ is feared that diabetes mellitus comorbidity 
will hinder the achievement of the long-term goal of eliminating TB. TB will be considered eliminated if by the year 2050 there is less than one incident case of the disease per one million population. ${ }^{3} 10$

Most of the scientific knowledge regarding association between diabetes and TB has been generated in industrialised countries. ${ }^{11}$ However, recently China and India have made valuable contributions. ${ }^{12} 13$ Currently, the published literature suffers from certain limitations. The majority of studies are either cross-sectional, casecontrol or retrospective cohort studies. The ascertainment of patient's diabetic status is based on past records, and a substantial number of studies fail to control for important confounders. ${ }^{5}{ }^{14} \quad 15$ Furthermore, good quality evidence needs to be generated from developing countries which can help in addressing this problem. Pakistan has a high burden of TB and a rapidly growing population of diabetics. Data are scarce regarding the impact of diabetes on $\mathrm{TB}$ treatment outcome in Pakistan. Therefore, this prospective cohort study was conducted to determine the difference between treatment outcomes in patients with diabetes and pulmonary tuberculosis (PTB) and patients without diabetes and PTB and identify the determinants of treatment outcomes in patients of PTB with and without diabetes mellitus.

\section{COHORT DESCRIPTION}

The recruitment and enrolment of new adult cases of PTB that were diagnosed, registered and received complete treatment at Gulab Devi Chest Hospital (GDH), Lahore, began in October, 2013. The diagnosis of PTB, sputum smear positive and sputum smear negative, was made according to the definition given by the National Tuberculosis Control Program (NTP). ${ }^{16}$ According to NTP, a patient having one or two sputum samples positive for acid-fast bacillus (AFB) is labelled as sputum smear-positive PTB patient. If both sputum samples are found negative, an antibiotic course of 7 days is prescribed to the patient. After 7 days, based on doctors' assessment of the patient an X-ray chest, if required, is taken, which if compatible with active PTB helps to declare the patient sputum smear negative PTB. A new case was a patient with PTB, sputum smear positive or sputum smear negative, who had never taken TB drugs in the past or had taken TB drugs for $<4$ weeks in the past but was not registered with the NTP. An adult was a patient with PTB aged 15 years or older. ${ }^{16}$ Patients with a history of antituberculosis treatment (ATT), who were not sure or were unable to recall previous therapy with ATT and who were severely ill, disabled or mentally ill were excluded from the study. All eligible patients with PTB willing to participate after giving informed written consent were enrolled in the study cohort.

The enrolment period lasted up to March 2014, until our sample size of 614 participants was successfully achieved. We recruited 614 patients with PTB on the basis of our statistical calculations for cohort studies using WHO software for sample size calculation in health studies. ${ }^{17}$ The diabetic status of enrolled patients with PTB was ascertained. The patients with diabetes and PTB and the patients without diabetes and PTB were followed-up prospectively during standardised category I treatment consisting of fixed dose combinations for adults in accordance with current guidelines of NTP. ${ }^{18}$ The intensive phase of treatment was of 2 months in which rifampicin, isoniazid, ethambutol and pyrazinamide were given to all patients on a daily basis. This was followed by a continuation phase of 4 months in which a daily dose of isoniazid and rifampicin was given to all patients.

\section{FOLLOW-UP}

The two groups comprising exposed (diabetic) PTB patients and unexposed (non-diabetic) patients were followed-up prospectively at second, fifth and sixth months of ATT treatment and also at 6 months after treatment completion. The follow-up schedule was thoroughly explained to respondents at recruitment, and the follow-up visits were scheduled to coincide with patients' drug collection time from GDH. At the time of recruitment, patients' contact details were gathered which included home addresses and two telephone numbers (landline or mobile) belonging either to them or a family member or a neighbour to facilitate the follow-up process. Follow-up reminders were sent through telephone calls. The contact information of the respondents was reviewed at each subsequent visit to aid in efficient follow-up. The follow-up period was from December 2013 to March 2015.

The first follow-up was scheduled at 2 months after the recruitment. The follow-up completion rate at this first follow-up was $93.4 \%$, with 40 participants lost to follow-up. The total number of participants entering the second follow-up was 558 patients with PTB instead of 574 , as 16 participants experienced a treatment outcome (13 deaths and 3 transferred out patients). The follow-up completion rate for the second phase was $93.3 \%$ with 37 patients with PTB lost to follow-up. For the third phase, the completion rate was $93 \%$ and 34 patients were lost to follow-up. The follow-up completion rate of the fourth phase was $100 \%$; however, the overall follow-up completion rate of the 614 patients with PTB was $81.9 \%$ with 111 patients lost to follow-up. Figure 1 highlights the number of respondents lost to follow-up at each phase of the study and also the number who experienced a treatment outcome during and at the end of ATT and also after treatment completion.

The common reasons for lost to follow-up included patients movement to another city, patients in denial of their disease status, patients attributing their signs and symptoms to black magic/supernatural power, patients claiming that they were incorrectly diagnosed and the 


\section{Prospective cohort study}

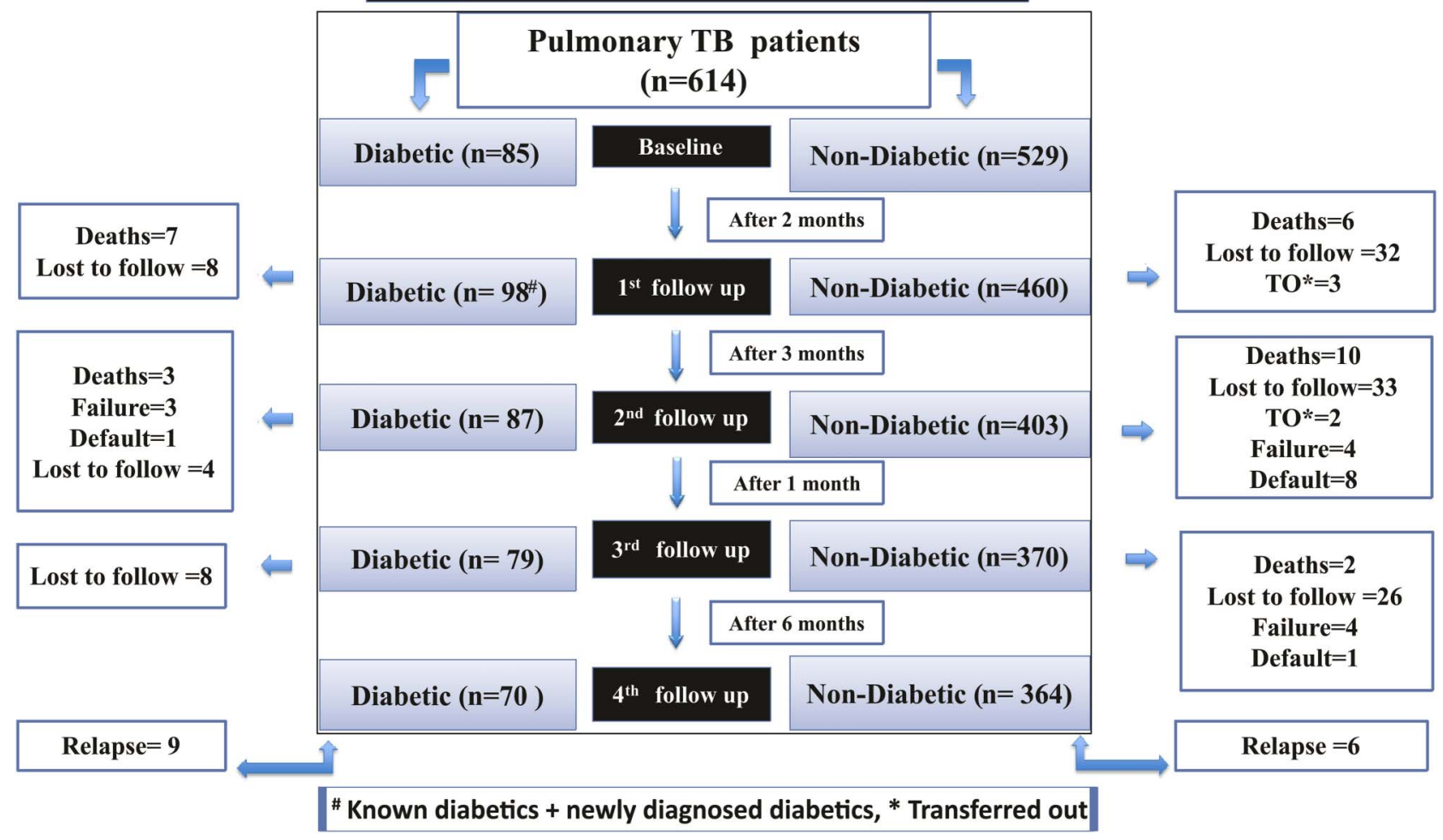

Figure 1 Flow diagram depicting the follow-up periods of the PTB cohort along with treatment outcome and loss to follow-up at Gulab Devi Chest Hospital, Lahore. TB, tuberculosis; PTB, pulmonary tuberculosis.

inability to contact the patient through telephone calls and home visits.

\section{ADDITIONAL DATA Interview}

Trained male and female data collectors conducted the interviews. They were employed as full-time data collectors who worked 6 days a week in the out patient department (OPD) and directly observed treatment, shortcourse (DOTS). Data were collected on structured questionnaires from the study participants at the time of recruitment and at each follow-up visit. The variables studied included sociodemographic characteristics such as age, gender, education, occupation, income, area of residence and marital status. Lifestyle and behavioural characteristics that included smoking status, alcohol consumption status, history of imprisonment, body mass index (BMI) and drug abuse were also determined. Patients' clinical presentation variables included cough longer than 3 weeks, prolonged fever, difficulty in breathing, presence of blood in sputum, night sweats, weight loss and type of PTB. Furthermore, history of comorbidities such as hypertension, heart disease, renal disease and asthma was inquired. Other variables studied were type of diabetes, glycaemic control among the diabetics, family history of diabetes, exposure to a household TB contact and adherence to DOTS therapy.
Information on these variables was collected at baseline and at each subsequent visit. Additionally, at each follow-up patients were inquired about adverse effects related to ATT, and data were collected on favourable and unfavourable outcomes among them.

\section{Measurement of height and weight}

The height of the patients with PTB was measured with a stadiometer to the last complete $0.1 \mathrm{~cm}$ and rounded to the nearest whole number while patients were standing erect without shoes. The weight of these patients was measured on a standing scale with minimal clothing on to the last $0.1 \mathrm{~kg}$ and rounded to the nearest whole number. Throughout the study, the same instruments were used which were calibrated every day to ensure the validity of the results. ${ }^{19}$

\section{Estimation of blood sugar}

Patients were inquired about their diabetic status, and those who had a known diagnosis of diabetes (selfreport) were labelled as diabetic. In those with unknown diabetic status, a random blood glucose (RBG) test was conducted at baseline and a fasting blood glucose (FBG) test was conducted at the first follow-up visit, which coincided with the second month of ATT treatment (figure 2). The protocol used to screen patients with $\mathrm{TB}$ for diabetes in China and India has been adhered to in this study. ${ }^{20-22}$ 
Figure 2 The protocol used in screening patients with PTB for diabetes. PTB, pulmonary tuberculosis.

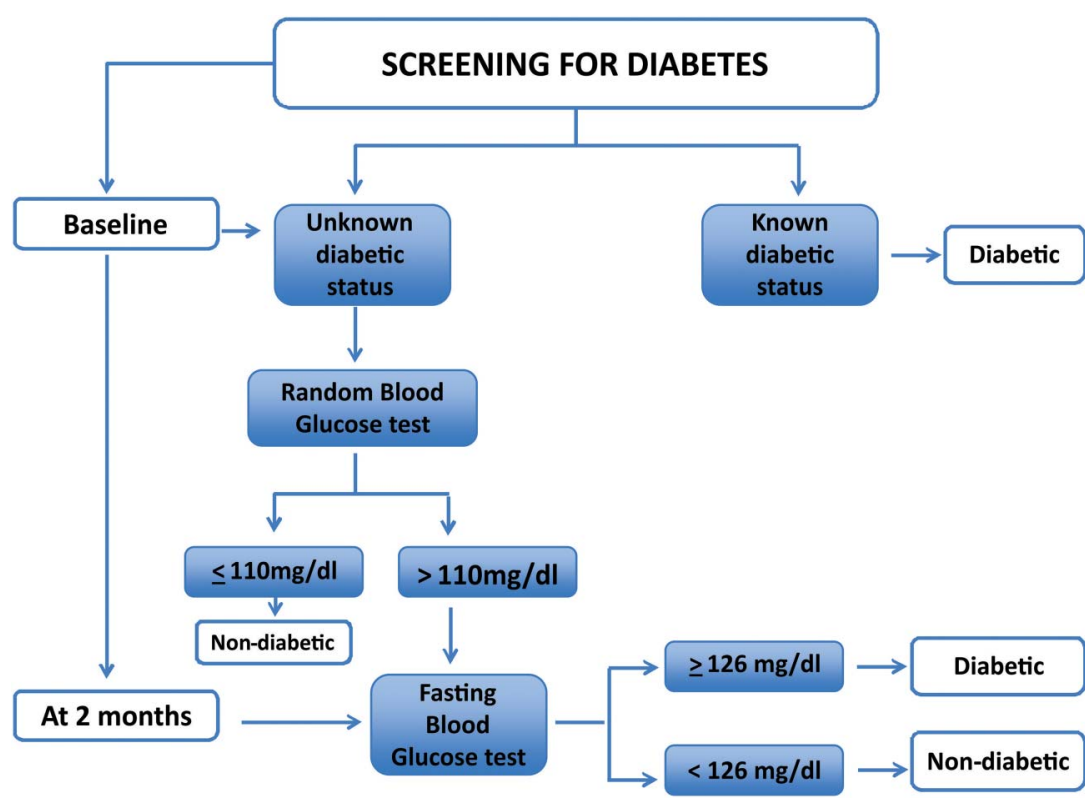

\section{RBG test}

Patients' RBG was estimated using the Accu-Check Active glucometer by Roche. All patients having a RBG above the cut-off value were offered FBG testing.

\section{FBG test}

Patients were asked to fast overnight, that is, 8 hours before coming for their first follow-up visit at the second month of ATT. They were reminded of the fast through telephone calls twice before their arrival at the health facility; first call a week before their date of visit to the hospital and the second call a day before the visit to ensure their eligibility for the FBG test. For FBG estimation, the same procedure mentioned above was carried out. However, before conducting the test, the patients were again inquired regarding their overnight fast to confirm their fasting status. In case of any suspicion of food intake, the patient was asked to come the next day for the FBG test. Diagnosis of diabetes was based on WHO guidelines. ${ }^{23}$ The diabetics were referred to a specialist for the management of their newly diagnosed disease. $^{22} 24$

\section{Sputum smear examination}

The diagnosis of patients with PTB and assessment of treatment outcomes were established by performing sputum smear microscopy using the Ziehl-Neelson (ZN) staining technique by the laboratory staff of GDH under the guidelines of NTP. The presence of AFB was checked in the sputum sample after preparing a sputum smear on a glass slide followed by its staining and fixation. The reporting of AFB was performed as depicted in table 1.

The results of the sputum sample were communicated by the laboratory personnel to OPD staff, who recorded it on the patients' treatment card and in hospitals' TB registers. The results were acquired by the data

\begin{tabular}{|c|c|c|c|}
\hline Seen on slide & Result & $\begin{array}{l}\text { Positive } \\
\text { (grading) }\end{array}$ & $\begin{array}{l}\text { Bacterial } \\
\text { load }\end{array}$ \\
\hline $\begin{array}{l}\text { More than } 10 \text { AFB per } \\
\text { field }\end{array}$ & POS & $3+$ & Heavy \\
\hline 1-10 AFB per field & POS & $2+$ & Medium \\
\hline $10-99$ AFB in 100 fields & POS & $1+$ & Low \\
\hline $1-9$ AFB in 100 fields & POS & $\begin{array}{l}\text { Record } \\
\text { actual } \\
\text { number }\end{array}$ & Very low \\
\hline No AFB in 100 fields & NEG & 0 & $\begin{array}{l}\mathrm{Nil} / \text { not } \\
\text { seen }\end{array}$ \\
\hline
\end{tabular}

collectors of the study from the patient treatment card and were verified with the hospital TB registers.

\section{Estimation of glycosylated haemoglobin}

At the second follow-up visit which coincided with the fifth month of ATT, a blood sample was drawn from all known and the newly diagnosed patients with diabetes and PTB for the estimation of gylcosylated haemoglobin. The glycaemic assessment was performed at a time when the effect of transient hyperglycaemia due to TB disease was probably negligible. This would provide an unbiased estimate of the association between patients' glycaemic control and treatment outcome. A 3 cc sample of blood was obtained through venipuncture by the data collectors using a disposable syringe and an aseptic technique. The blood was immediately transferred to a tube containing ethylenediaminetetraacetic acid, which was gently inverted to ensure the mixing of the components. The specimen was transported on a daily basis to the pathology laboratory of Punjab Institute of Cardiology 
(PIC), where the samples were analysed for haemoglobin A1c (HbA1c) using high-performance liquid chromatography (HPLC). A HbAlc value of $\leq 7 \%$ was considered to be a normal value, and a HbAlc value $>7 \%$ was considered to be an abnormal value in the study. ${ }^{25}$

\section{TREATMENT OUTCOMES}

All the patients were followed-up to determine treatment outcomes. The standardised treatment outcome definitions given by the NTP, Pakistan, and the WHO were followed in the study. ${ }^{26}$ The treatment outcomes are as follows.

Cured: A sputum smear positive patient, who had completed 6 months of treatment and became sputum smear negative at the end of treatment and on at least one previous occasion.

Treatment completed: A sputum smear positive patient, who completed 6 months of treatment and had at least one follow-up smear negative result and none at the end of treatment due to any reason or smear negative cases who completed 6 months of treatment successfully.

Death: A patient who died for any reason during the course of treatment.

Failure: A sputum smear positive patient who remained positive or again became positive at 5 months or a sputum smear negative patient found to be smear positive at the end of 2 months.

Figure 3 Flow diagram of data collection activity at Gulab Devi Hospital, Lahore.
Default: A patient whose treatment was interrupted for two consecutive months or more after registration (according to new definition, this treatment outcome is called 'loss to follow-up').

Transferred out: A patient who was transferred to another centre and for whom the treatment outcome was not known (according to new definition, this treatment outcome is called 'not evaluated').

Relapse: A patient who was previously treated for $\mathrm{TB}$ was declared cured or treatment completed at the end of their treatment and was diagnosed with a recurrent episode of TB (either a true relapse or a new episode of TB caused by reinfection). ${ }^{27}$

Treatment outcomes were categorised into favourable and unfavourable treatment outcomes. The 'unfavourable outcome' included patients who defaulted, died, were transferred out, had treatment failure and had relapse. The category of 'favourable outcome' included patients who were cured and completed treatment.

The various data collection activities undertaken by the DITTO study are depicted in figure 3 .

\section{CHARACTERISTICS OF THE STUDY POPULATION}

Of the total 661 new PTB patients who fulfilled the inclusion criteria, 614 patients consented to participate in the study, whereas 47 refused participation. The 
Table 2 Profile of 614 new pulmonary tuberculosis patients with $(n=113)$ or without diabetes mellitus $(n=501)$ presenting at Gulab Devi Chest Hospital, Lahore

\begin{tabular}{|c|c|c|c|c|c|c|}
\hline & \multicolumn{2}{|c|}{$\begin{array}{l}\text { PTB with diabetes } \\
n=113\end{array}$} & \multicolumn{2}{|c|}{$\begin{array}{l}\text { PTB without } \\
\text { diabetes } \\
n=501\end{array}$} & \multirow{2}{*}{$\begin{array}{l}\text { Total } \\
\mathrm{n}=614 \\
\mathrm{n}(\%)\end{array}$} & \multirow[b]{2}{*}{ p value } \\
\hline & $\mathrm{n}$ & Percent & $\bar{n}$ & Percent & & \\
\hline Age group (in years) & & & & & & $<0.001$ \\
\hline $15-19$ & 1 & 1 & 134 & 27 & $135(22)$ & \\
\hline $20-24$ & 4 & 3.5 & 138 & 27 & $142(23)$ & \\
\hline $25-29$ & 4 & 3.5 & 63 & 13 & $67(11)$ & \\
\hline $30-39$ & 16 & 14 & 74 & 15 & $90(15)$ & \\
\hline $40-49$ & 30 & 27 & 37 & 7 & $67(11)$ & \\
\hline$>50$ & 58 & 51 & 55 & 11 & $113(18)$ & \\
\hline Gender & & & & & & 0.357 \\
\hline Male & 53 & 47 & 259 & 52 & $312(51)$ & \\
\hline Female & 60 & 53 & 242 & 48 & $302(49)$ & \\
\hline Sputum smear status & & & & & & 0.232 \\
\hline Positive & 67 & 59 & 266 & 53 & $333(54)$ & \\
\hline Negative & 46 & 41 & 235 & 47 & $281(46)$ & \\
\hline Residence & & & & & & 0.179 \\
\hline Urban & 84 & 74 & 340 & 68 & $424(69)$ & \\
\hline Rural & 29 & 26 & 161 & 32 & $190(31)$ & \\
\hline Educational qualification & & & & & & 0.035 \\
\hline Illiterate & 74 & 65.5 & 249 & 50 & $323(52)$ & \\
\hline Primary & 13 & 11.5 & 71 & 14 & $84(14)$ & \\
\hline Matriculation & 20 & 18 & 126 & 25 & $146(24)$ & \\
\hline Intermediate & 5 & 4 & 25 & 5 & $30(5)$ & \\
\hline Bachelors & 1 & 1 & 17 & 3 & $18(3)$ & \\
\hline Masters and above & 0 & 0 & 13 & 3 & $13(2)$ & \\
\hline Income category (rupees) & & & & & & 0.113 \\
\hline $\mathrm{Nil}^{\star}$ & 73 & 65 & 311 & 62 & $384(63)$ & \\
\hline$<5000$ & 5 & 5 & 38 & 8 & $43(7)$ & \\
\hline 5100-8000 & 6 & 5 & 61 & 12 & $67(11)$ & \\
\hline $8100-11000$ & 10 & 9 & 44 & 9 & $54(9)$ & \\
\hline $11100-14000$ & 7 & 6 & 19 & 4 & $26(4)$ & \\
\hline $14100-17000$ & 6 & 5 & 15 & 3 & $21(3)$ & \\
\hline$>17100$ & 6 & 5 & 13 & 2 & $19(3)$ & \\
\hline Marital status & & & & & & $<0.001$ \\
\hline Married & 101 & 89 & 243 & 48.4 & $344(56)$ & \\
\hline Single & 12 & 11 & 255 & 51 & 267 (43.5) & \\
\hline Divorced & 0 & 0 & 1 & 0.2 & $1(0.2)$ & \\
\hline Widowed & 0 & 0 & 2 & 0.4 & $2(0.3)$ & \\
\hline BMI† & & & & & & $<0.001$ \\
\hline$<18.50$ & 18 & 17 & 289 & 58 & $307(51)$ & \\
\hline $18.50-24.99$ & 63 & 58 & 194 & 39 & $257(42)$ & \\
\hline 25-29.99 & 18 & 17 & 9 & 2 & $27(4)$ & \\
\hline 30 and above & 9 & 8 & 8 & 1 & $17(3)$ & \\
\hline Heart disease & & & & & & $<0.001$ \\
\hline Yes & 14 & 12 & 2 & 0.4 & $16(3)$ & \\
\hline No & 99 & 88 & 499 & 99.6 & $598(97)$ & \\
\hline Hypertension & & & & & & $<0.001$ \\
\hline Yes & 26 & 23 & 13 & 3 & $39(6)$ & \\
\hline No & 87 & 77 & 488 & 97 & $575(94)$ & \\
\hline
\end{tabular}

assessment of 614 respondents' diabetic status revealed that $113(18 \%)$ were diabetic and $501(82 \%)$ were non-diabetic. The age distribution of the PTB cohort comprising of $113(18 \%)$ diabetics and $501(82 \%)$ non- diabetics shows the respondents with diabetes and PTB to be older (mean age $=47.4$ ) as compared to the respondents without diabetes and PTB (mean age=28.5) $(p<0.001)$. A greater proportion of patients with 
diabetes and PTB were illiterate $(\mathrm{n}=74 / 113,65.5 \%)$ as compared to patients without diabetes and PTB $(\mathrm{n}=249 / 501,50 \%) \quad(\mathrm{p}=0.035)$. The majority of patients with diabetics and PTB were married $(n=101 / 113,89 \%)$ as opposed to patients without diabetes and PTB $(\mathrm{n}=243 / 501,58 \%) \quad(\mathrm{p}<0.001)$. The majority of the exposed patients with PTB were overweight $(n=18 / 113$, $17 \%)$ and obese $(n=9 / 113,8 \%)$, whereas, the majority of unexposed respondents were underweight $(n=289$ / $501,58 \%)(\mathrm{p}<0.001)$. More patients with diabetes and PTB gave a history of heart disease $(\mathrm{n}=14 / 113,12 \%)$ and hypertension $(n=26 / 113,23 \%)$ as compared to patients without diabetes and PTB $(\mathrm{n}=2 / 501,0.4 \%$ and $\mathrm{n}=13501,3 \%)(\mathrm{p}<0.001)$, respectively (table 2$)$.

\section{FINDINGS TO DATE}

The treatment outcome analysed as a binary variable shows that $69(14 \%)$ patients had an unfavourable outcome and $434(86 \%)$ had a favourable outcome. In univariate logistic regression analysis, patients with diabetes were more likely to experience an unfavourable outcome than patients without diabetes $(\mathrm{OR}=2.6,95 \%$ CI 1.48 to $4.56, \mathrm{p}=0.001)$. Other studies conducted in Taiwan and South Korea have also reported an increased risk of unfavourable treatment outcome among patients with diabetes and PTB as compared to patients without diabetes and PTB, that is, an OR of 1.46 (95\% CI of 1.03 to 2.08$)^{28}$ and 1.78 (95\% CI 1.07 to 2.95$),{ }^{29}$ respectively.

\section{STRENGTHS AND LIMITATIONS}

The strength of our study is a rigorous study design, that is, the prospective cohort study design, which generates valid results as opposed to other observational epidemiological study designs in our endeavour to determine treatment outcomes among patients with diabetes and TB. The data collection tool gathered information on all possible confounders identified through literature review and having biological plausibility. These confounders will thus be adjusted for in the analysis producing valid results. The exposure status of patients with PTB was based on two tests; one random and the other FBG test. The confirmatory FBG test was conducted 2 months after the initiation of ATT to rule out the bias associated with transient stress-induced hyperglycaemia attributed to TB. Finally, standardised treatment outcome definitions provided by WHO were followed in the study. To the best of our knowledge, no previous study has been conducted in Pakistan to determine the effect of diabetes on the treatment outcome of TB patients.

The study found it beneficial to employ a male data collector and a female data collector who were trained for gender-matched data collection considering the prevailing cultural environment. We ensured negligible data collector turnover, which helped develop a good rapport between the researcher and respondents. Additionally, we provided a 24-hour helpline, which was very popular among the patients. It was greatly appreciated by them and helped develop sustained relationships with them, thus maximising our response rate.

However, there were certain limitations in our study. The drug susceptibility testing was not carried out among the PTB cohort at the time of enrolment or during the course of ATT, which could have led to bias in the results. However, because of our inclusion criteria of recruiting only the new patients with PTB with no prior history of ATT intake, drug resistance may not be an issue. The difference in drug resistance patterns between the two groups was unlikely to have contributed to the observed results. Second, HIV status which has been identified as a strong risk factor for adverse treatment outcome among patients with $\mathrm{TB}$ was not determined. Finally, we were unable to study the effect of glucose control on TB treatment outcome as HbA1c values for the entire cohort were not available. Owing to resource constraints, glycosylated haemoglobin blood analyses were performed only on the diabetics in the study. If treatment outcome among patients with diabetes and PTB is modified by glucose control, our results could be affected. However, according to Mi et $a l^{30}{ }^{2}$ and 6 month FBG levels among patients with PTB did not have statistically significant association with adverse outcomes.

Contributors FM contributed to conception and design of the work, acquisition, analysis and interpretation of data and write-up. ZAB contributed to conception of work, analysis of data, revised the work for intellectual content and approved the final version to be published.

\section{Competing interests None declared.}

Ethics approval Ethical approval was obtained from the Institutional Ethical Review Committee of Health Services Academy, Islamabad, on 17th September 2013 (F. No. 107/2013-IERC/HSA). Permission was also taken from the administration of the Gulab Devi Chest Hospital, Lahore, where data collection was undertaken. All patients gave written informed consent before recruitment in the study.

Provenance and peer review Not commissioned; externally peer reviewed.

Data sharing statement The data are not available freely, however we welcome specific and detailed proposals for collaboration. Enquiries and requests for further information should be made to fatimamukhtar@doctor. com.

Open Access This is an Open Access article distributed in accordance with the Creative Commons Attribution Non Commercial (CC BY-NC 4.0) license, which permits others to distribute, remix, adapt, build upon this work noncommercially, and license their derivative works on different terms, provided the original work is properly cited and the use is non-commercial. See: http:// creativecommons.org/licenses/by-nc/4.0/

\section{REFERENCES}

1. Dooley KE, Chaisson RE. Tuberculosis and diabetes mellitus: convergence of two epidemics. Lancet Infect Dis 2009;9:737-46.

2. Jimenez-Corona ME, Cruz-Hervert LP, Garcia-Garcia L, et al. Association of diabetes and tuberculosis: impact on treatment and post-treatment outcomes. Thorax 2013;68:214-20.

3. Brostrom RJ. Summary of the impact of diabetes on tuberculosis control and Submission of draft standards for diabetes and tuberculosis in the US-affiliated Pacific Islands, Meeting Paper: 6. Fifth Pacific Stop TB Meeting. 4-7 May 2010. Nadi, Fiji Islands. 
4. Restrepo BI, Fisher-Hoch SP, Smith B, et al. Mycobacterial clearance from sputum is delayed during the first phase of treatment in patients with diabetes. Am J Trop Med Hyg 2008;79:541-4.

5. Baker MA, Harries AD, Jeon CY, et al. The impact of diabetes on tuberculosis treatment outcomes: a systematic review. BMC Med 2011;9:81.

6. World Health Organization. WHO report 2011. Global Tuberculosis Control. Geneva: WHO, 2011.

7. World Health Organization. Global tuberculosis report 2015. 20th ed. France: World Health Organization; 2015.

8. Restrepo BI, Camerlin AJ, Rahbar $\mathrm{MH}$, et al. Cross-sectional assessment reveals high diabetes prevalence among newly-diagnosed tuberculosis cases. Bull WHO 2011;89: 352-9.

9. Hakeem R, Fawwad A. Diabetes in Pakistan: epidemiology, determinants and prevention. Inter J Diabetol 2011;3:4.

10. World Health Organisation. Collaborative framework for care and control of tuberculosis and diabetes. Geneva: WHO, Stop TB Department, 2011.

11. Wang CS, Yang CJ, Chen HC, et al. Impact of type 2 diabetes on manifestations and treatment outcome of pulmonary tuberculosis. Epidemiol Infect 2009;137:203-10.

12. Lin Y, Li L, Mi F, et al. Screening patients with diabetes mellitus for tuberculosis in China. Trop Med Int Health 2012;17:1302-8.

13. Indian Diabetes Mellitus-Tuberculosis Study Group. Screening of patients with diabetes mellitus for tuberculosis in India. Trop Med Int Health 2013;18:646-54.

14. Sullivan T, Ben Amor Y. The co-management of tuberculosis and diabetes: challenges and opportunities in the developing world. PLoS Med 2012;9:e1001269.

15. Jeon CY, Murray MB. Diabetes mellitus increases the risk of active tuberculosis: a systematic review of 13 observational studies. PLoS Med 2008;5:e152.

16. Tuberculosis Control Programme Pakistan. Doctors Training course on community-based TB care-DOTS. Department for International Development (DFID), World Health Organisation (WHO), 2012.

17. Lwanga SK, Lemeshow S. Sample size determination in health studies-a practical manual. Software version by KC Lun, P Chiam.
Software version by the National University of Singapore. Geneva: 1991; World Health Organization.

18. Ministry of Health, Government of Pakistan. Refresher module for doctors. Provincial TB control programme Punjab, 2008.

19. Dodor EA. Evaluation of nutritional status of new tuberculosis patients at the effia-nkwanta regional hospital. Ghana Med J 2008;42:22-8

20. Lin Y, Li L, Mi F, et al. Screening patients with tuberculosis for diabetes mellitus in China. Trop Med Int Health 2012;17:1294-301.

21. Indian Diabetes Mellitus-Tuberculosis Study Group. Screening of patients with tuberculosis for diabetes mellitus in India. Trop Med Int Health 2013;18:636-45.

22. Prakash BC, Ravish KS, Prabhakar B, et al. Tuberculosis-diabetes mellitus bidirectional screening at a tertiary care centre, South India. Public Health Action 2013;3:S18-22.

23. World Health Organisation. Definition and diagnosis of diabetes mellitus and intermediate hyperglycemia. Geneva: WHO/ International Diabetes Federation, 2006.

24. Raghraman S, Vasudevan KP, Govindarajan S, et al. Prevalence of diabetes mellitus among tuberculosis patients in Urban Puducherry. N Am J Med Sci 2014;6:30-4.

25. Balasubramanian R, Ramanathan U, Thyagarajan K, et al. Evaluation of an intermittent six-month regimen in new pulmonary tuberculosis patients with diabetes mellitus. Indian $J$ Tuberc 2007;54:168-76.

26. Desk Guide for Doctors on Management of Tuberculosis. National Tuberculosis Control Program, Pakistan, 2015

27. World Health Organisation. Definitions and reporting framework for tuberculosis-2013 revised. Geneva: WHO, Stop TB Department, 2013.

28. Chiang $\mathrm{CY}$, Bai KJ, Lin $\mathrm{HH}$, et al. The influence of diabetes, glycemic control, and diabetes-related comorbidities on pulmonary tuberculosis. PLoS One 2015;10:e0121698.

29. Choi $\mathrm{H}$, Lee $\mathrm{M}$, Chen $\mathrm{RY}$, et al. Predictors of pulmonary tuberculosis treatment outcomes in South Korea: a prospective cohort study, 2005-2012. BMC Infect Dis 2014;14:360.

30. Mi F, Tan S, Liang L, et al. Diabetes mellitus and tuberculosis: pattern of tuberculosis, two-month smear conversion and treatment outcomes in Guangzhou, China. Trop Med Int Health 2013;18:1379-85. 